\title{
A MAXIMUM PRINCIPLE
}

\author{
KUNG-FU NG
}

(Received 25 March 1977)

Communicated by A. P. Robertson

\begin{abstract}
Let $K$ be a nonempty compact set in a Hausdorff locally convex space, and $F$ a nonempty family of upper semicontinuous convex-like functions from $K$ into $[-\infty, \infty) . K$ is partially ordered by $F$ in a natural manner. It is shown among other things that each isotone, upper semicontinuous and convex-like function $g: K \rightarrow[-\infty, \infty)$ attains its $K$-maximum at some extreme point of $K$ which is also a maximal element of $K$.
\end{abstract}

Subject classification (Amer. Math. Soc. (MOS) 1970): primary 46 A 40.

Let $K$ be a nonempty compact set in a Hausdorff locally convex topological vector space $E$ and $F$ a nonempty family of convex, upper semi-continuous functions from $K$ into $[-\infty, \infty)$. Recall that a function $f$ on $K$ is convex if

$$
f\left(\lambda_{1} k_{1}+\lambda_{2} k_{2}\right) \leqslant \lambda_{1} f\left(k_{1}\right)+\lambda_{2} f\left(k_{2}\right)
$$

whenever $0 \leqslant \lambda_{1}, \lambda_{2} \leqslant 1, \lambda_{1}+\lambda_{2}=1$ and $k_{1}, k_{2}, \lambda_{1} k_{1}+\lambda_{2} k_{2}$ are in $K$, and that $f$ is said to be affine if both $f$ and $-f$ are convex. Also $K$ has a natural quasi-ordering induced by $F$ :

$x \leqslant y$ if and only if $f(x) \leqslant f(y)$ for all $f \in F$.

We write $x \sim y$ if $x \leqslant y$ and $y \leqslant x$. An element $x$ of $K$ is said to be maximal if $y \sim x$ whenever $x \leqslant y$. The $F$-boundary of $K$ is, by definition, the set of all maximal extreme points of $K$ and will be denoted by $\partial_{F} K$. In the special case when $K$ is convex and each $f$ in $F$ is affine, a generalized Bauer's maximum principle proved by Lumer (1963) and Edwards (1970) asserts that each $f$ in $F$ attains its $K$-maximum on $\partial_{F} K$. In some situations one wishes to have a similar principle applicable to certain non-affine, even non-convex functions. For example, if $K$ is taken to be the closed unit disc $\Delta$ in the complex plane and $F$ the set $S(\Delta)$ of all continuous functions $f$ on 
$\Delta$ such that $f$ is subharmonic on the interior of $\Delta$, then $\partial_{F} \Delta$ is precisely the topological boundary $\partial \Delta$, that is, the circumference of $\Delta$; hence, by the classical maximum principle (see Conway (1973), p. 266), each $f$ in $F$ attains its $\Delta$-maximum on the $F$-boundary $\partial_{F} \Delta$ of $\Delta$, though $f$ may not be convex on $\Delta$. In this note, we extend the above theorem of Lumer and Edwards to the case when $K$ and $f$ in $F$ are not necessarily convex.

Recall first that a non-empty subset $A$ of $K$ is extreme if $x, y \in A$ whenever $\lambda x+(1-\lambda) y \in A$ for some $\lambda \in(0,1)$ and $x, y \in K$. A function $f: K \rightarrow[-\infty, \infty)$ is said to be convex-like if, for each closed extreme subset $A$ of $K$, the set

$$
\{a \in A: f(a)=\sup f(A)\}
$$

is either empty or else an extreme subset of $A$. Thus, each convex function is certainly convex-like; also each function $f$ in $S(\Delta)$ is convex-like on $\Delta$ because proper extreme subsets of $\Delta$ are the sets contained in $\partial \Delta$. From now on we shall assume that $F$ is a nonempty family of upper semi-continuous and convex-like functions from a compact (not necessarily convex) set $K$ in $E$ into $[-\infty, \infty)$ and that $K$ is ordered by $\leqslant$ induced by $F$. An extended real-valued function $g$ on $K$ is said to be isotone if $g(x) \leqslant g(y)$ whenever $x \leqslant y$ in $K$. For example, each $f$ in $F$ is isotone; more generally, if $g$ is the limit function of a pointwise convergent net in $F$, then $g$ is isotone.

THEOREM 1. Each isotone, upper semi-continuous and convex-like function $g: K \rightarrow[-\infty, \infty)$ attains its $K$-maximum on the $F$-boundary $\partial_{F} K$ of $K$.

Proof. Let $\mathscr{E}$ be the collection of all nonempty extreme, compact and increasing subsets of $K$ (a subset $A$ of $K$ is increasing if $k \in A$ whenever $a \leqslant k$ for some $a \in A$ ). Since $K \in \mathscr{E}, \mathscr{E}$ is a non-empty set, partially ordered by set inclusion. By Zorn's Lemma, each member of $\mathscr{E}$ contains a minimal member of $\mathscr{E}$. By assumption on $g$, the set

$$
G=\{x \in K: g(x)=\sup g(K)\}
$$

is a member of $\mathscr{E}$ and hence contains a minimal member, say $Q$ of $\mathscr{E}$. Since $Q$ is a compact extreme subset of $K, Q$ contains at least one extreme point $x$ of $K$ by virtue of the Krein-Milman theorem. It remains to show that $x$ is maximal in the quasi-ordered set $K$. For a contradiction, let us assume that there exists $y$ in $K$ such that $x \leqslant y$ but $y \leqslant x$. Then $y \in Q$ since $Q$ is increasing, and there exists $f$ in $F$ such that $f(y)>f(x)$. Notice that the set

$$
\{z \in Q: f(z)=\sup f(Q)\}
$$

is a member of $\mathscr{E}$, properly contained in $Q$. This contradicts the minimality of $Q$. 
The following theorem was proved by Bauer in the special case when $K$ is convex.

THEOREM 2. Let $K$ be a compact subset of $E$. Then each upper semi-continuous convex function $f: K \rightarrow[-\infty, \infty)$ attains its $K$-maximum on the extreme boundary $\partial_{e} K$ of $K$.

Indeed, if we take $F$ to consist of all upper semi-continuous convex functions on $K$, then the partial ordering induced by $F$ is simply equality and $\partial_{e} K=\partial_{F} K$.

An immediate consequence of Theorem 2 is the following strong version of the Krein-Milman theorem: each compact subset $K$ in $E$ is contained in the closed convex hull of its extreme points. Moreover, Theorem 1 may also be used to prove the following generalization of Dini's theorem.

TheOREM 3. Let $\left\{g_{i}: i \in I\right\}$ be a downward directed family of isotone, upper semicontinuous convex functions on $K$ into $[0, \infty)$ such that $\lim _{i} g_{i}(x)=0$ for each $x$ in the F-boundary $\partial_{F} K$. Then $\left\{g_{i}\right\}$ converges to 0 uniformly on $K$.

Proof. Let $g_{0}$ denote the limit function of $\left\{g_{i}\right\}$. Then $g_{0}$ satisfies the conditions in Theorem 1 and $g_{0} \geqslant 0$ on $K$ with equality on $\partial_{F} K$. By Theorem 1, we must have $g_{0}=0$ on $K$. Consequently, it follows from the classical theorem of Dini that $\left\{g_{i}\right\}$ converges to $g_{0}=0$ uniformly on $K$.

Finally, we note an interesting application of Theorem 3 to the theory of ordered vector spaces. Let $V$ be a partially ordered normed space with a normal cone $C$ and $\left(V^{\prime}, C^{\prime}\right)$ the partially ordered Banach dual space (with the natural dual ordering). Let $K=\left\{v^{\prime} \in C^{\prime}:\left\|v^{\prime}\right\| \leqslant 1\right\}$. Then $K$ is a compact convex subset of $V^{\prime}$ under the $\sigma\left(V^{\prime}, V\right)$-topology. Let $F$ be the set of all continuous affine functions on $K$ of the form

$$
\tilde{x}: v^{\prime} \rightarrow\left\langle x, v^{\prime}\right\rangle,
$$

where $x \in C$. Then the ordering in $K$ induced by $F$ is simply the dual ordering. Moreover, since $C$ is normal, the Krein-Grosberg theorem (see Wong and $\mathrm{Ng}$ (1973)) asserts that if $\left\{v_{i}: i \in I\right\}$ is a directed upward family of elements in $V$ and if $v \in V$ is such that $\lim _{i}\left\langle v_{i}, v^{\prime}\right\rangle=\left\langle v, v^{\prime}\right\rangle$ for each $v^{\prime}$ in $K$ then $v=\sup _{i} v_{i}$ and $\lim _{i}\left\|v_{i}-v\right\|=0$.

The following theorem extends this result.

THEOREM 4. Let $\left\{v_{i}\right\}$ be a directed upward family of elements in $V$ and $v \in V$ be such that $v_{i} \leqslant v$ for each $i$. Suppose that $\lim _{i}\left\langle v_{i}, v^{\prime}\right\rangle=\left\langle v, v^{\prime}\right\rangle$ for each $v^{\prime}$ in $\partial_{F} K$. Then $v=\sup _{i} v_{i}$ and $\lim _{i}\left\|v_{i}-v\right\|=0$. 
ProOF. Let $g_{i}=\tilde{v}-\tilde{v}_{i}$. Applying Theorem 3, we conclude that

$$
\lim _{i}\left\langle\boldsymbol{v}_{i}, v^{\prime}\right\rangle=\left\langle v, v^{\prime}\right\rangle
$$

for each $v^{\prime} \in K$.

REMARK. In Schaefer (1974), p. 89, it is inferred that the condition $v_{i} \leqslant v$ for all $i$ can be dropped. Unfortunately this in fact is not correct. For example, let $V=l_{1}$. Then it is easily verified that $\partial_{F} K$ is a singleton consisting of $e=(1,1, \ldots) \in l_{\infty}=V^{\prime}$. For each $n$, let

$$
v_{n}=\left(1, \frac{1}{2}, \ldots \frac{1}{2^{n}}, 0,0, \ldots\right) \in l_{1} .
$$

Then $\lim _{n}\left\langle v_{n}, e\right\rangle=\langle v, e\rangle$ where $v=(2,0,0, \ldots)$, say; but $\lim _{n}\left\|v_{n}-v\right\| \neq 0$.

\section{References}

J. B. Conway (1973), Functions of one complex variable (Graduate texts in mathematics, Springer-Verlag, Berlin).

D. A. Edwards (1970), 'An extension of Choquet boundary theory to certain partially ordered compact convex sets', Studia Math. 36, 177-193.

G. Lumer (1963), 'Points extrémeaux associés; frontiéres de Shilov et Choquet; principe du minimum', C. R. Acad. Sc. Paris 256, 858-861.

Y. C. Wong and K. F. Ng (1973), Partially ordered topological vector spaces (Clarendon Press, Oxford).

H. H. Schaefer (1974), Banach lattices and positive operators (Springer-Verlag, Berlin).

Mathematics Department

Science Centre

The Chinese University of Hong Kong

Shatin, N.T.

Hong Kong 